\title{
Evidence of systemic endothelial injury and microthrombosis in hospitalized COVID-19 patients at different stages of the disease
}

\author{
Domenico G. Della Rocca ${ }^{1}$ Michele Magnocavallo ${ }^{2}$ Carlo Lavalle ${ }^{2}$ Jorge Romero ${ }^{3}$. Giovanni B. Forleo ${ }^{4}$. \\ Nicola Tarantino ${ }^{3}$. Cristina Chimenti ${ }^{2}$. Isabella Alviz ${ }^{3}$. Maria T. Gamero ${ }^{3}$ Mario J. Garcia ${ }^{3} \cdot$ Luigi Di Biase $^{3}$. \\ Andrea Natale ${ }^{1}$
}

Accepted: 30 October 2020 / Published online: 6 November 2020

(c) Springer Science+Business Media, LLC, part of Springer Nature 2020

\section{Highlights}

- Schistocytes are fragments of red blood cells which may be encountered in the peripheral blood smear of patients suffering from a variety of microangiopathic diseases.

- In the hospitalized COVID-19 patients at different stages of disease severity, a schistocyte count $\geq 1 \%$ was documented in approximately $70 \%$ of patients.

- Evidence of myocardial injury was observed in $87.5 \%$ of all who had a count of schistocytes $\geq 1 \%$.

- Schistocytes may serve as a simple and inexpensive biomarker to identify a high-risk subpopulation with a latent systemic microvascular damage irrespective of respiratory symptoms.

Severe endothelial injury and widespread microthrombosis have been recently described in postmortem examinations of coronavirus disease-2019 (COVID-19) patients [1-5].

Whether a systemic microangiopathy is present at different stages of the disease irrespective of the extent of pulmonary involvement, it has not been confirmed.

Yet, a wide range of extrapulmonary clinical manifestations (e.g. thromboembolism, myocardial infarction with

Domenico G. Della Rocca and Michele Magnocavallo have contributed equally to this work.

Michele Magnocavallo

michele.magnocavallo@uniroma1.it

St. David's Medical Center, Austin, USA

2 Department of Clinical, Internal, Anesthesiology and Cardiovascular Sciences, Policlinico Universitario

Umberto I, Sapienza University of Rome, Viale del

Policlinico, 155- 00161 Rome, Italy

3 Montefiore Medical Center, Bronx, NY, USA

4 Azienda Ospedaliera-Universitaria Luigi Sacco, Milan, Italy normal coronary arteries, kidney function impairment) has been reported in a significant number of COVID-19 patients [6]. Such manifestations usually accompany the most common respiratory symptoms, even if they appear to be unrelated to the severity of lung involvement, and may manifest at any stage of the disease, therefore suggesting the presence of an underlying systemic vascular disorder with hypercoagulability as a common pathophysiological substrate of the disease.

Schistocytes are fragments of red blood cells which may be encountered in the peripheral blood smear (PBS) of patients suffering from a variety of microangiopathic diseases [e.g. disseminated intravascular coagulation (DIC), thrombocytopenic purpura].

They are the result of a mechanical damage to erythrocytes which are sheared by fibrin strands of microthrombi in the peripheral circulation. The underlying pro-thrombotic state is linked to a damage of the endothelium which promotes thrombus formation and microvascular dysfunction.

In this context, the presence of schistocytes may serve as a surrogate biomarker for in-vivo assessment of a diffuse endothelial damage with formation of fibrin thrombi.

We aimed at documenting the presence of schistocytes in the PBS of hospitalized COVID-19 patients at different stages of disease severity.

Fourteen consecutive patients with severe acute respiratory syndrome coronavirus- 2 infection confirmed by reversetranscriptase-polymerase-chain-reaction were included in this study. The median age was 70 years (IQR: 59-76) and $85.7 \%$ were males. Baseline characteristics are reported in Table 1.

PBSs were taken after a median of 3 days from admission (range 1-5 d) and examined by two experienced pathologists who were blinded to disease severity. The presence of schistocytes (abnormal cut-off value $\geq 1 \%$ ) was microscopically evaluated following the International Council for Standardization in Hematology recommendations [7]. 
Table 1 Baseline characteristics, laboratory results, drug therapy, and outcomes of the overall population (Panel A), and characteristics of 7 patients requiring mechanical ventilation (Panel B) and of 7 patients under noninvasive ventilation or high-flow nasal cannula (Panel C)

\begin{tabular}{|c|c|c|c|c|c|c|c|c|}
\hline \multicolumn{3}{|l|}{ Characteristic } & \multicolumn{2}{|c|}{ Overall $(n=14)$} & \multicolumn{2}{|c|}{$\begin{array}{l}\text { Invasive Mechanical Ventilation } \\
(\mathrm{n}=7)\end{array}$} & \multicolumn{2}{|c|}{$\begin{array}{l}\text { Noninvasive Ventilation/ } \\
\text { Nasal Cannula }(\mathrm{n}=7)\end{array}$} \\
\hline \multicolumn{9}{|l|}{ Panel A } \\
\hline \multicolumn{3}{|c|}{ Age, median (IQR) [yr] } & \multicolumn{2}{|c|}{$70(59-76)$} & \multicolumn{2}{|c|}{$70(58-76)$} & \multicolumn{2}{|l|}{$70(60-75)$} \\
\hline \multicolumn{3}{|c|}{ Male Sex, n (\%) } & \multicolumn{2}{|c|}{$12(85.7)$} & \multicolumn{2}{|l|}{$7(100)$} & \multicolumn{2}{|l|}{$5(71.4)$} \\
\hline \multicolumn{9}{|l|}{ Medical History } \\
\hline \multicolumn{3}{|c|}{ Hypertension, n (\%) } & \multicolumn{2}{|c|}{$10(71.4)$} & \multicolumn{2}{|c|}{$5(71.4)$} & \multicolumn{2}{|l|}{$5(71.4)$} \\
\hline \multicolumn{3}{|c|}{ Diabetes, n (\%) } & \multicolumn{2}{|c|}{$8(57.1$} & \multicolumn{2}{|c|}{$5(71.4)$} & \multicolumn{2}{|l|}{$3(42.9)$} \\
\hline Symptoms at $\mathrm{O}$ & & & & & & & & \\
\hline Fever, n (\%) & & & 2.9) & & $7(100)$ & & $6(85.7)$ & \\
\hline Cough, n (\%) & & & 78.6) & & $5(71.4$ & & $6(85.7)$ & \\
\hline Diarrhea, n ( $\%$ & & & 1.4) & & $1(14.3$ & & $2(28.6)$ & \\
\hline Imaging Featur & & & & & & & & \\
\hline Ground-Glass & pacity, n (\%) & & 2.9) & & $7(100)$ & & $6(85.7)$ & \\
\hline Bilateral Puln & nary Infiltrates, $\mathrm{n}($ & & 100) & & $7(100)$ & & $7(100)$ & \\
\hline Laboratory Fin & $\operatorname{lgs}$ & & & & & & & \\
\hline White-Cell C & $\mathrm{nt} / \mathrm{mm}^{3}$, median $(\mathrm{IC}$ & (R) & $0(5,880-10,900)$ & & 6,870 & $0-12,600)$ & $6,200(5,950$ & $, 050)$ \\
\hline White-Cell C & $\mathrm{nt} / \mathrm{mm}^{3}>10,000, \mathrm{n}$ & $(\%)$ & 3.6) & & $3(42.9$ & & $1(14.3)$ & \\
\hline Lymphocytes & ount $/ \mathrm{mm}^{3}$, median & (IQR) & $(667-1,052)$ & & $790(68$ & ,140) & $700(545-95$ & \\
\hline Lymphocytes & ount $/ \mathrm{mm}^{3}<1,000$ & $n(\%)$ & 4.3) & & $4(57.1)$ & & $5(71.4)$ & \\
\hline Platelet Coun & $\mathrm{nm}^{3}$, median $(\mathrm{IQR})$ & & $000(150,000-245$ & 00) & 163,00 & 06,000-196,000) & $\begin{array}{l}229,000(19 \\
341,00)\end{array}$ & 00 \\
\hline Platelet Coun & $\mathrm{nm}^{3}<100,000, \mathrm{n}(9$ & & 4.3) & & $2(28.6$ & & $0(0.0)$ & \\
\hline LDH, median & QR) [U/L] & & $(284-531)$ & & $295(27$ & 19) & $398(353-42$ & \\
\hline $\mathrm{LDH}>280 \mathrm{U}$ & $\mathrm{n}(\%)$ & & 71.4) & & $4(57.1)$ & & $6(85.7)$ & \\
\hline Creatinine, $\mathrm{m}$ & $\operatorname{ian}(\mathrm{IQR})[\mu \mathrm{g} / \mathrm{L}]$ & & 33-135) & & $84(82-$ & & $117(97-146$ & \\
\hline PT, median (I & R) $[\mathrm{sec}]$ & & (12.3-16.8) & & $12.4(1$ & $-13.8)$ & $15.0(12.7-2$ & \\
\hline aPTT, median & IQR) [sec] & & (31.5-35.8) & & $34.1(3$ & $-48.9)$ & $32.0(29.9-3$ & \\
\hline Fibrinogen, $m$ & $\operatorname{lian}(\mathrm{IQR})[\mathrm{g} / \mathrm{L}]$ & & $2.8-7.26)$ & & $2.9(1.6$ & & $6.3(4.6-7.9)$ & \\
\hline Fibrinogen $<$ & /L, n (\%) & & & & $0(0.0)$ & & $0(0.0)$ & \\
\hline D-dimer, med & n (IQR) [mg/L] & & $(1.26-4.47)$ & & $2.21(1$ & $-4.47)$ & $1.21(0.91-3$ & \\
\hline D-dimer $>1 \mathrm{n}$ & $/ \mathrm{L}, \mathrm{n}(\%)$ & & 78.6) & & $7(100)$ & & $4(57.1)$ & \\
\hline Peripheral Bloo & Smear & & & & & & & \\
\hline Schistocyte > & $\%, \mathrm{n}(\%)$ & & 1.4) & & $5(71.4$ & & $5(71.4)$ & \\
\hline Treatment & & & & & & & & \\
\hline Antibiotic Ag & $\mathrm{t}, \mathrm{n}(\%)$ & & 2.9) & & $6(85.7)$ & & $7(100)$ & \\
\hline Antiviral Age & $\mathrm{n}(\%)$ & & & & $1(14.3$ & & $0(0.0)$ & \\
\hline Hydroxychlor & fuine, n (\%) & & $2.9)$ & & $6(85.7)$ & & $7(100)$ & \\
\hline Corticosteroic & $\mathrm{n}(\%)$ & & 3.6) & & $1(14.3)$ & & $3(42.9)$ & \\
\hline Outcomes & & & & & & & & \\
\hline Death, n (\%) & & & .4) & & $2(28.6$ & & $1(14.3)$ & \\
\hline Characteristic & Patient 1 & Patient 2 & Patient 3 & Pat & & Patient 5 & Patient 6 & Patient 7 \\
\hline Panel B & & & & & & & & \\
\hline Age, yr & 72 & 82 & 70 & 54 & & 38 & 81 & 62 \\
\hline Sex & Male & Male & Male & Ma & & Male & Male & Male \\
\hline Initial findings & & & & & & & & \\
\hline $\begin{array}{l}\text { Medical his- } \\
\text { tory }\end{array}$ & $\begin{array}{l}\text { Hypertension } \\
\text { Diabetes Hyper- } \\
\text { thyroidism }\end{array}$ & $\begin{array}{l}\text { Hypertensior } \\
\text { Diabetes } \\
\text { Alzheimer }\end{array}$ & Hypertension & $\begin{aligned} \mathrm{Hy} \\
\mathrm{D}\end{aligned}$ & $\begin{array}{l}\text { tension } \\
\text { etes }\end{array}$ & Asthma & $\begin{array}{l}\text { Hypertension } \\
\text { Diabetes }\end{array}$ & Diabetes \\
\hline
\end{tabular}


Table 1 (continued)

\begin{tabular}{|c|c|c|c|c|c|c|c|}
\hline Characteristic & Patient 1 & Patient 2 & Patient 3 & Patient 4 & Patient 5 & Patient 6 & Patient 7 \\
\hline $\begin{array}{l}\text { Symptoms at } \\
\text { disease onset }\end{array}$ & $\begin{array}{l}\text { Fever } \\
\text { Dyspnea } \\
\text { Cough } \\
\text { Diarrhea }\end{array}$ & $\begin{array}{l}\text { Fever } \\
\text { Dyspnea } \\
\text { Syncope }\end{array}$ & $\begin{array}{l}\text { Fever } \\
\text { Dyspnea } \\
\text { Cough }\end{array}$ & $\begin{array}{l}\text { Fever } \\
\text { Dyspnea } \\
\text { Cough }\end{array}$ & $\begin{array}{l}\text { Fever } \\
\text { Dyspnea } \\
\text { Cough }\end{array}$ & $\begin{array}{l}\text { Fever } \\
\text { Dyspnea } \\
\text { Cough }\end{array}$ & $\begin{array}{l}\text { Fewer } \\
\text { Dyspnea } \\
\text { Asthenia }\end{array}$ \\
\hline $\begin{array}{l}\text { Imaging } \\
\text { features }\end{array}$ & $\begin{array}{l}\text { Ground-glass } \\
\text { opacity, } \\
\text { Bilateral pulmo- } \\
\text { nary infiltrates }\end{array}$ & $\begin{array}{l}\text { Ground-glass } \\
\text { opacity, } \\
\text { Bilateral } \\
\text { pulmonary } \\
\text { infiltrates }\end{array}$ & $\begin{array}{l}\text { Ground-glass } \\
\text { opacity, } \\
\text { Bilateral pulmo- } \\
\text { nary infiltrates }\end{array}$ & $\begin{array}{l}\text { Ground-glass } \\
\text { opacity, } \\
\text { Bilateral pulmo- } \\
\text { nary infiltrates }\end{array}$ & $\begin{array}{l}\text { Ground-glass } \\
\text { opacity, } \\
\text { Bilateral pulmo- } \\
\text { nary infiltrates }\end{array}$ & $\begin{array}{l}\text { Ground-glass } \\
\text { opacity, } \\
\text { Bilateral } \\
\text { pulmonary } \\
\text { infiltrates }\end{array}$ & $\begin{array}{l}\text { Ground- } \\
\text { glass } \\
\text { opacity, } \\
\text { Bilateral } \\
\text { pulmo- } \\
\text { nary } \\
\text { infil- } \\
\text { trates }\end{array}$ \\
\hline Treatment & $\begin{array}{l}\text { Hydroxychloro- } \\
\text { quine Antibi- } \\
\text { otics }\end{array}$ & $\begin{array}{l}\text { Hydroxy- } \\
\text { chloroquine } \\
\text { Lopinavir- } \\
\text { Ritonavir }\end{array}$ & $\begin{array}{l}\text { Hydroxychloro- } \\
\text { quine Immune } \\
\text { Globulin } \\
\text { Antibiotics }\end{array}$ & $\begin{array}{l}\text { Hydroxychloro- } \\
\text { quine Antibi- } \\
\text { otics }\end{array}$ & $\begin{array}{l}\text { Immune Globulin } \\
\text { Antibiotics }\end{array}$ & $\begin{array}{l}\text { Hydroxy- } \\
\text { chloroquine } \\
\text { Immune } \\
\text { Globulin } \\
\text { Antibiotics }\end{array}$ & $\begin{array}{l}\text { Hydroxy- } \\
\text { chloro- } \\
\text { quine } \\
\text { Immune } \\
\text { Globulin } \\
\text { Antibiot- } \\
\text { ics }\end{array}$ \\
\hline \multicolumn{8}{|l|}{$\begin{array}{l}\text { Laboratory find- } \\
\text { ings }\end{array}$} \\
\hline $\begin{array}{c}\text { White cell } \\
\text { count } \\
\text { (per mm3) }\end{array}$ & 6,610 & 23,290 & 4,970 & 6,870 & 13,740 & 11,470 & 3,920 \\
\hline $\begin{array}{l}\text { Total Neutro- } \\
\text { phils } \\
\text { (per mm3) }\end{array}$ & 5,370 & 21,800 & 3,740 & 4,750 & 11,910 & 7,210 & 3,070 \\
\hline $\begin{array}{l}\text { Total Lym- } \\
\text { phocytes } \\
\text { (per mm3) }\end{array}$ & 710 & 790 & 660 & 1,210 & 1,070 & 3,670 & 330 \\
\hline $\begin{array}{l}\text { Total mono- } \\
\text { cytes } \\
\text { (per mm3) }\end{array}$ & 270 & 260 & 400 & 320 & 540 & 340 & 360 \\
\hline $\begin{array}{l}\text { Platelet count } \\
\text { (per mm3) }\end{array}$ & 217,000 & 361,000 & 163,000 & 140,000 & 65,000 & 175,000 & 75,000 \\
\hline $\begin{array}{l}\text { Hemoglobin } \\
(\mathrm{g} / \mathrm{L})\end{array}$ & 88 & 103 & 137 & 88 & 112 & 89 & 83 \\
\hline Albumin $(\mathrm{g} / \mathrm{L})$ & 25 & 26 & 34 & 36 & 30 & 26 & 27 \\
\hline $\begin{array}{l}\text { Alanine ami- } \\
\text { notransferase } \\
\text { (U/L) }\end{array}$ & 23 & 25 & 22 & 14 & 72 & 62 & 22 \\
\hline $\begin{array}{l}\text { Aspartate ami- } \\
\text { notransferase } \\
\text { (U/L) }\end{array}$ & 20 & 40 & 35 & 12 & 5 & 25 & 9 \\
\hline $\begin{array}{l}\text { Lactate dehy- } \\
\text { drogenase } \\
\text { (U/L) }\end{array}$ & 249 & 295 & 280 & 383 & 1,433 & 654 & 276 \\
\hline $\begin{array}{l}\text { Creatinine } \\
(\mathrm{mg} / \mathrm{dL})\end{array}$ & 1.55 & 1.10 & 0.81 & 0.71 & 0.84 & 0.95 & 0.83 \\
\hline $\begin{array}{l}\text { High-sensitiv- } \\
\text { ity cardiac } \\
\text { troponin } \mathrm{T} \\
(\mu \mathrm{g} / \mathrm{L})\end{array}$ & 0.042 & 0.073 & 0.010 & 0.009 & 0.033 & 0.009 & 0.046 \\
\hline $\begin{array}{l}\text { Prothrombin } \\
\text { time }(\mathrm{sec})\end{array}$ & 11.07 & 12.41 & 12.34 & 11.49 & 14.93 & 12.75 & 26.62 \\
\hline
\end{tabular}


Table 1 (continued)

\begin{tabular}{|c|c|c|c|c|c|c|c|}
\hline Characteristic & Patient 1 & Patient 2 & Patient 3 & Patient 4 & Patient 5 & Patient 6 & Patient 7 \\
\hline $\begin{array}{l}\text { Activated par- } \\
\text { tial thrombo- } \\
\text { plastin time } \\
(\mathrm{sec})\end{array}$ & 34.1 & 32.1 & 71.0 & 35.6 & 29.6 & 32.5 & 62.2 \\
\hline $\begin{array}{l}\text { Fibrinogen } \\
(\mathrm{g} / \mathrm{L})\end{array}$ & 8.39 & 6.67 & 1.96 & 3.61 & 1.18 & 2.88 & 1.12 \\
\hline $\begin{array}{l}\text { D-Dimer } \\
(\mathrm{mg} / \mathrm{L})\end{array}$ & 4.473 & 2.217 & 1.886 & 1.430 & 4.474 & 4.502 & 1.732 \\
\hline $\begin{array}{l}\text { Procalcitonin } \\
\text { (ng/mL) }\end{array}$ & 0.04 & 0.88 & 0.05 & 0.18 & 0.16 & 0.22 & 0.023 \\
\hline $\begin{array}{l}\text { High-sensi- } \\
\text { tivity }\end{array}$ & 3.3 & 318 & 1.4 & 27.5 & 3.2 & 10.9 & 46.4 \\
\hline \multicolumn{8}{|l|}{$\begin{array}{l}\text { C-reactive pro- } \\
\text { tein }(\mathrm{mg} / \mathrm{L})\end{array}$} \\
\hline \multicolumn{8}{|l|}{$\begin{array}{l}\text { Peripheral blood } \\
\text { smear }\end{array}$} \\
\hline $\begin{array}{l}\text { Schistocyte } \\
(\%)\end{array}$ & $1-2$ & $1-2$ & $1-2$ & $1-2$ & 0.5 & 0.5 & $1-2$ \\
\hline Characteristic & Patient 8 & Patient 9 & Patient 10 & Patient 11 & Patient 12 & Patient 13 & Patient 14 \\
\hline \multicolumn{8}{|l|}{ Panel C } \\
\hline Age, yr & 75 & 82 & 70 & 76 & 42 & 63 & 58 \\
\hline Sex & Male & Male & Female & Male & Male & Female & Male \\
\hline \multicolumn{8}{|l|}{ Initial findings } \\
\hline $\begin{array}{l}\text { Medical his- } \\
\text { tory }\end{array}$ & Hypertension & $\begin{array}{l}\text { Hypertension } \\
\text { COPD }\end{array}$ & $\begin{array}{l}\text { Hypertension } \\
\text { Diabetes }\end{array}$ & $\begin{array}{l}\text { Hypertension } \\
\text { Diabetes }\end{array}$ & - & $\begin{array}{l}\text { Hypertension } \\
\text { Diabetes }\end{array}$ & - \\
\hline $\begin{array}{l}\text { Symptoms } \\
\text { at disease } \\
\text { onset }\end{array}$ & $\begin{array}{l}\text { Fever } \\
\text { Dyspnea } \\
\text { Cough } \\
\text { Diarrhea }\end{array}$ & $\begin{array}{l}\text { Fever } \\
\text { Dyspnea } \\
\text { Cough } \\
\text { Diarrhea }\end{array}$ & $\begin{array}{l}\text { Fever } \\
\text { Dyspnea } \\
\text { Cough }\end{array}$ & $\begin{array}{l}\text { Dyspnea } \\
\text { Cough }\end{array}$ & $\begin{array}{l}\text { Fever } \\
\text { Dyspnea } \\
\text { Cough }\end{array}$ & $\begin{array}{l}\text { Fever } \\
\text { Dyspnea } \\
\text { Cough }\end{array}$ & $\begin{array}{l}\text { Fever } \\
\text { Dyspnea }\end{array}$ \\
\hline $\begin{array}{l}\text { Imaging } \\
\text { features }\end{array}$ & $\begin{array}{l}\text { Ground-glass } \\
\text { opacity, } \\
\text { bilateral pulmo- } \\
\text { nary infiltrates }\end{array}$ & $\begin{array}{l}\text { Ground-glass } \\
\text { opacity, } \\
\text { bilateral pulmo- } \\
\text { nary infiltrates }\end{array}$ & $\begin{array}{l}\text { Focal airspace } \\
\text { disease in the } \\
\text { peripheral } \\
\text { right midlung, } \\
\text { discoid atelec- } \\
\text { tasis at the left } \\
\text { lung base }\end{array}$ & $\begin{array}{l}\text { Ground-glass } \\
\text { opacity, } \\
\text { bilateral } \\
\text { pulmonary } \\
\text { infiltrates }\end{array}$ & $\begin{array}{l}\text { Ground-glass } \\
\text { opacity, } \\
\text { bilateral } \\
\text { pulmonary } \\
\text { infiltrates }\end{array}$ & $\begin{array}{l}\text { Ground-glass } \\
\text { opacity, } \\
\text { bilateral } \\
\text { pulmonary } \\
\text { infiltrates }\end{array}$ & $\begin{array}{l}\text { Ground-glass } \\
\text { opacity, } \\
\text { bilateral pulmo- } \\
\text { nary infiltrates }\end{array}$ \\
\hline Treatment & $\begin{array}{l}\text { Hydroxychloro- } \\
\text { quine immune } \\
\text { globulin } \\
\text { antibiotics }\end{array}$ & $\begin{array}{l}\text { Hydroxychloro- } \\
\text { quine immune } \\
\text { globulin } \\
\text { antibiotics }\end{array}$ & $\begin{array}{l}\text { Hydroxy- } \\
\text { chloroquine } \\
\text { antibiotics }\end{array}$ & $\begin{array}{l}\text { Hydroxy- } \\
\text { chloroquine } \\
\text { antibiotics }\end{array}$ & $\begin{array}{l}\text { Hydroxy- } \\
\text { chloroquine } \\
\text { antibiotics }\end{array}$ & $\begin{array}{l}\text { Hydroxy- } \\
\text { chloroquine } \\
\text { antibiotics }\end{array}$ & $\begin{array}{l}\text { Hydroxychloro- } \\
\text { quine antibiot- } \\
\text { ics }\end{array}$ \\
\hline \multicolumn{8}{|l|}{$\begin{array}{l}\text { Laboratory find- } \\
\text { ings }\end{array}$} \\
\hline $\begin{array}{l}\text { White cell } \\
\text { count } \\
\text { (per mm3) }\end{array}$ & 2,750 & 5,800 & 8,830 & 6,250 & 11,670 & 6,120 & 9,380 \\
\hline $\begin{array}{l}\text { Total neutro- } \\
\text { phils } \\
\text { (per mm3) }\end{array}$ & 1,470 & 5,010 & 7,400 & 7,500 & 2,000 & - & 8,200 \\
\hline $\begin{array}{l}\text { Total lym- } \\
\text { phocytes } \\
\text { (per mm3) }\end{array}$ & 690 & 350 & 900 & 400 & 700 & 1,000 & 1,500 \\
\hline $\begin{array}{l}\text { Total mono- } \\
\text { cytes } \\
\text { (per mm3) }\end{array}$ & 410 & 270 & 300 & 500 & 400 & 200 & 200 \\
\hline
\end{tabular}


Table 1 (continued)

\begin{tabular}{|c|c|c|c|c|c|c|c|}
\hline Characteristic & Patient 8 & Patient 9 & Patient 10 & Patient 11 & Patient 12 & Patient 13 & Patient 14 \\
\hline $\begin{array}{l}\text { Platelet } \\
\text { count (per } \\
\text { mm3) }\end{array}$ & 146,000 & 165,000 & 430,000 & 229,000 & 221,000 & 251,000 & 531,000 \\
\hline $\begin{array}{l}\text { Hemoglobin } \\
(\mathrm{g} / \mathrm{L})\end{array}$ & 112 & 127 & 98 & 128 & 169 & 96 & 137 \\
\hline $\begin{array}{l}\text { Albumin } \\
(\mathrm{g} / \mathrm{L})\end{array}$ & 30 & 28 & 31 & 36 & 47 & 30 & 33 \\
\hline $\begin{array}{l}\text { Alanine ami- } \\
\text { notrans- } \\
\text { ferase } \\
\text { (U/L) }\end{array}$ & 110 & 23 & 10 & 24 & 48 & 23 & 144 \\
\hline $\begin{array}{l}\text { Aspartate } \\
\text { ami- } \\
\text { notrans- } \\
\text { ferase } \\
\text { (U/L) }\end{array}$ & 151 & 12 & 20 & 59 & 75 & 47 & 36 \\
\hline $\begin{array}{l}\text { Lactate } \\
\text { dehydro- } \\
\text { genase (U/ } \\
\text { liter) }\end{array}$ & 357 & 398 & 241 & 348 & 506 & 677 & 539 \\
\hline $\begin{array}{l}\text { Creatinine } \\
(\mathrm{mg} / \mathrm{dL})\end{array}$ & 0.72 & 0.98 & 1.61 & 0.97 & 1.35 & 1.50 & 0.67 \\
\hline $\begin{array}{l}\text { High- } \\
\text { sensitivity } \\
\text { cardiac } \\
\text { troponin } \mathrm{T} \\
(\mu \mathrm{g} / \mathrm{L})\end{array}$ & 0.009 & 0.012 & 0.1 & 0.076 & 0.037 & 0.011 & 0.023 \\
\hline $\begin{array}{l}\text { Prothrombin } \\
\text { time (sec) }\end{array}$ & 12.55 & 10.76 & 35.9 & 13.2 & - & 16.8 & 33.1 \\
\hline $\begin{array}{l}\text { Activated } \\
\text { partial } \\
\text { thrombo- } \\
\text { plastin time } \\
(\mathrm{sec})\end{array}$ & 29.9 & 32 & - & 36.2 & 35.4 & - & 13.2 \\
\hline $\begin{array}{l}\text { Fibrinogen } \\
(\mathrm{g} / \mathrm{L})\end{array}$ & 2.80 & 4.26 & 7.46 & 6.27 & 4.97 & 8.39 & 8.59 \\
\hline $\begin{array}{r}\text { D-Dimer } \\
(\mathrm{mg} / \mathrm{L})\end{array}$ & 0.909 & 4.481 & 1.940 & 0.920 & 1.210 & 4.930 & 0.810 \\
\hline $\begin{array}{l}\text { Procalcitonin } \\
\text { (ng/mL) }\end{array}$ & 0.08 & 0.08 & 0.5 & 0.1 & 0.09 & 0.3 & 0.01 \\
\hline \multicolumn{8}{|l|}{$\begin{array}{l}\text { C-reactive } \\
\text { protein } \\
(\mathrm{mg} / \mathrm{L})\end{array}$} \\
\hline \multicolumn{8}{|l|}{$\begin{array}{l}\text { Peripheral blood } \\
\text { smear }\end{array}$} \\
\hline $\begin{array}{l}\text { Schistocyte } \\
(\%)\end{array}$ & $0.5-1$ & 0 & $1-2$ & $1-2$ & $1-2$ & $1-2$ & $1-2$ \\
\hline
\end{tabular}

aPTT Activated partial thromboplastin time, $I Q R$ interquartile range, $L D H$ lactate dehydrogenase, $P T$ prothrombin

Patients with mechanical cardiac valvular prostheses, chronic kidney disease stage 4-5, diabetic microangiopathy, or other causes of schistocyte formation were excluded.
None had required hemodialysis or extracorporeal membrane oxygenation during hospitalization.

Symptoms and signs at presentation included: fever (93\%), cough (79\%), and diarrhea (21\%). 
At the time PBS was performed, patients were hospitalized and had different degrees of COVID-19 severity: $7(50 \%)$ patients had severe lung injury requiring invasive mechanical ventilation, 2 (14.3\%) noninvasive ventilation, and $5(35.7 \%)$ high-flow nasal cannula.

A schistocyte count $\geq 1 \%$ was documented in $10(71.4 \%)$ patients; one $(7.1 \%)$ patient had $0.8 \%$ and $3(21.4 \%)$ had $\leq 0.5 \%$.

The median platelet count was $196,000 / \mathrm{mm}^{3}$ (IQR: $150,000-245,000)$ and all but $2(14.3 \%)$ patients had $>100,000$ platelets $/ \mathrm{mm}^{3}$.

None had a fibrinogen level $<1 \mathrm{~g} / \mathrm{L}$ and fulfilled the diagnostic criteria for overt DIC.

Evidence of myocardial injury, as demonstrated by elevated levels of high-sensitive troponin T $(>0.014 \mu \mathrm{g} / \mathrm{L})$, was observed in $8(57.1 \%)$ patients, 7 of whom $(87.5 \%)$ had a count of schistocytes $\geq 1 \%$ and no preexisting history of cardiovascular disease. All 8 patients had normal left ventricular ejection fraction (EF) but one (patient 2) with reduced $\mathrm{EF}$ and regional wall motion abnormalities.

All patients were prescribed with systemic anticoagulation, $4(28.6 \%)$ received low-dose corticosteroids.

During hospitalization, one patient had pulmonary thromboembolism (patient 10$)$ and $3(21.4 \%)$ died of multiorgan failure. All four patients had a count of schistocytes $>1 \%$. At the time of discharge, PBS was repeated in other 4 patients with a previous abnormal schistocyte value [after a median of 22 days (range 16-28)], revealing a normal count in all.

Hereby, we report a high prevalence (71.4\%) of an abnormal count of schistocytes in the PBS of COVID-19 patients. Schistocytes were observed at any stage of disease severity, irrespective of lung involvement. Additionally, increased high-sensitive troponin $\mathrm{T}$ was observed in the majority of patients with schistocytes ( 7 out of 10; 70.0\%), compared to those without.

Since none of the patients fulfilled the diagnostic criteria for overt DIC and other causes of schistocyte formation were excluded, the presence of these fragments of red blood cells may imply a subclinical impairment of the endothelial cell layer of the microvasculature with formation of microthrombi in the coronary and peripheral circulation.

These findings are consistent with recent studies which described endotheliitis and a systemic microthrombotic disease in patients who died from COVID-19 [1, 3]. However, our study for the first time extends the observations of postmortem studies by correlating a similar pathophysiological substrate also to milder forms of the disease.

This pattern of endothelial injury and hypercoagulability may explain the variety of clinical manifestations (e.g. kidney failure, myocardial infarction with normal coronary arteries, neurological manifestations, purpura) that has been described so far in COVID-19 patients [6].
As such, a therapeutic approach targeting the underlying endothelial dysfunction and prothrombotic state (e.g. early systemic anticoagulation, immunomodulators) may be justified at any stage of the disease to prevent clinical progression and multi-organ involvement.

Additionally, schistocytes may serve as a simple and inexpensive biomarker to identify a high-risk subpopulation with a latent systemic microvascular damage irrespective of respiratory symptoms.

Acknowledgements This letter is dedicated to all the Italian Healthcare workers on the frontline fighting SARS-CoV-2.

Funding None.

\section{Compliance with ethical standards}

Conflict of interest All other authors have reported that they have no relationships relevant to the contents of this paper to disclose.

\section{References}

1. Ackermann M, Verleden SE, Kuehnel M, Haverich A, Welte T, Laenger F et al (2020) Pulmonary Vascular Endothelialitis, Thrombosis, and Angiogenesis in Covid-19. N Engl J Med. https ://doi.org/10.1056/NEJMoa2015432

2. Schaller T, Hirschbühl K, Burkhardt K, Braun G, Trepel M, Märkl $B$ et al (2020) Postmortem Examination of Patients With COVID19. JAMA. https://doi.org/10.1001/jama.2020.8907

3. Varga Z, Flammer AJ, Steiger P, Haberecker M, Andermatt R, Zinkernagel AS et al (2020) Endothelial cell infection and endotheliitis in COVID-19. The Lancet 395:1417-1418. doi:https ://doi.org/10.1016/S0140-6736(20)30937-5

4. Della Rocca DG, Pepine CJ (2014) Some thoughts on the continuing dilemma of angina pectoris. Eur Heart J 35(21):1361-1364. doi:https://doi.org/10.1093/eurheartj/ehs225

5. Lowenstein CJ, Solomon SD (2020) Severe COVID-19 is a Microvascular Disease. Circulation. https://doi.org/10.1161/CIRCU LATIONAHA.120.050354

6. Wang D, Hu B, Hu C, Zhu F, Liu X, Zhang J et al (2020) Clinical Characteristics of 138 Hospitalized Patients With 2019 Novel Coronavirus-Infected Pneumonia in Wuhan, China. JAMA 323:1061. doi:https://doi.org/10.1001/jama.2020.1585

7. Zini G, d'ONOFRIO G, Briggs C, Erber W, Jou JM, Lee SH et al (2012) ICSH recommendations for identification, diagnostic value, and quantitation of schistocytes: ICSH RECOMMENDATIONS FOR DETECTION AND QUANTITATION OF SCHISTOCYTES. International Journal of Laboratory Hematology 34:107-116. doi:https://doi.org/10.1111/j.1751-553X.2011.01380

Publisher's Note Springer Nature remains neutral with regard to jurisdictional claims in published maps and institutional affiliations. 